\title{
Neel Effect toroidal current sensor
}

\author{
Eric Vourc' $h^{1}$, Yu Wang ${ }^{1}$, Pierre-Yves Joubert ${ }^{2}$, Bertrand Revol $^{1}$, André Couderette ${ }^{3}$, Lionel Cima ${ }^{3}$ \\ ${ }^{1}$ SATIE, ENS Cachan, CNRS, Universud, 94235 Cachan Cedex, France \\ ${ }^{2}$ IEF, Université de Paris-Sud, CNRS, 91405 Orsay, France \\ ${ }^{3}$ Neelogy S.A. 191, Avenue Aristide Briand 94230 Cachan France
}

In this paper, a configuration for a Neel effect AC-DC current sensor designed with second harmonic sensing in view is proposed. This configuration relies on the use of two toroidal superparamagnetic cores wound in opposite direction with respect to each other and connected in series. An analytical model is developed, which shows that the component of the output signal at the excitation frequency, which is useless, should be removed provided that the windings are symmetrical. The latter principle is validated experimentally and a prototype device is characterized for different excitation conditions. Open loop DC and AC current measurements are carried out over the $[-110 \mathrm{~A} 110 \mathrm{~A}]$ range.

Index Terms-Current Sensor, Electric current control, Magnetic sensor, Nanoparticles, Power system management, Superparamagnetism.

\section{INTRODUCTION}

$\mathrm{T}^{\mathrm{s}}$ he measurement of current is an important stake in particular for the energy conversion, the energy management and the energy storage systems. Many sensor technologies exist such as shunts, Hall effect probes, fluxgate sensors or Rogowski coils, which exhibit different features from one to another (for example regarding the sensitivity, the dynamic range, the bandwidth, the only $\mathrm{AC}$ or the both $\mathrm{AC}$ and DC operation, the size, the weight, the implementation facility,...) [1-4]. Neel effect current ${ }^{\circledR}$ sensors are based on a magnetic field transducer made up of magnetic nanoparticles embedded in a plastic matrix [5]. Due to the superparamagnetic (SPM) behavior of the nanoparticles [6] the transducer features a non-linear magnetization characteristic which also has the property of being remanence free. Advantage can be taken of this non-linearity in order to accurately measure currents over large ranges. On the other hand, likewise Rogowski coils, the possibility of building flexible Neel effect sensors thanks to the use of a flexible plastic matrix is also an advantage as it enables easy to install sensors to be designed. By superposing a sinusoidal magnetic field excitation to the DC or AC (slowly varying with respect to the excitation) magnetic field induced by the current to measure, a time varying magnetic flux density is sensed by the coil wound over the transducer. Thus, an electromotive force (EMF) appears at the output of the sensor. It has been demonstrated [7] that given the non linearity of the magnetic characteristic of the transducer the amplitude of the component of the EMF at the second order harmonic of the excitation frequency is an odd function of the current to measure. As this function is monotonous over a large magnetic field range the measurement of currents over ranges in the order of tens to thousands of Amperes can be envisaged, depending of the magnetization characteristic of the transducer.

Here, we report on a Neel effect sensor featuring a toroidal configuration and an excitation and measurement configuration using a single coil. This configuration is chosen so as to enable the EMF component at the excitation frequency $f_{e x}$ to be removed, and so that the output signal consists in the suitable component, that is that at the second harmonic of the excitation frequency.

This paper is organized as follows: firstly, in section II, the principle of generic Neel effect current sensors is reminded and an analytical expression of their output EMF is given. Secondly, the concept of double core toroidal sensors is reported and an analytical expression of their output signal is derived. In section III a prototype device is characterized for different excitation currents. Both the measurement of DC and AC currents are carried out, which show that with the used device open loop measurements of DC and AC currents can be performed over the $[-110 \mathrm{~A} 110 \mathrm{~A}]$ range.

\section{NEEl EFFEct CuRRENT SENSORS PRINCIPLE}

\section{A. Elementary Neel Effect Sensor}

Let us consider an elementary Neel effect sensor consisting in a SPM core wound with one sensing turn. An analytical model can be derived for such a sensor [7]. Consider that the latter sensor is submitted to both a $H_{m}$ magnetic field to measure and a sinusoidal excitation magnetic field $H_{e x}(t)=h_{e x} \sin \left(2 \pi f_{e x} t\right) \quad$ (which can be, for example, induced by a sinusoidal current feeding an excitation winding superposed to the measurement winding) both normal to the turn cross section and homogeneous over the cross section area. At any point of the sensing coil cross section the secondorder Taylor's expansion of the magnetic flux density $B$ centred at $H_{m}$ is

$$
B(H) \cong B\left(H_{m}\right)+\left.\left(H-H_{m}\right) \frac{\partial B}{\partial H}\right|_{H_{m}}+\left.\frac{\left(H-H_{m}\right)^{2}}{2} \frac{\partial^{2} B}{\partial H^{2}}\right|_{H_{m}}
$$

According to (1) and to Lenz's law and assuming that the magnetic field to measure $H_{m}$ can be considered as constant in time also for an AC waveform provided that its frequency is much lower than that of the excitation magnetic field, the EMF $\varepsilon(t)$ at the output of the elementary sensing coil writes 


$$
\begin{aligned}
\varepsilon(t)=-S \frac{\partial B(H)}{\partial t} \cong- & S \mu_{0} \mu_{r}\left(H_{m}\right) \omega_{e x} h_{e x} \cos \left(\omega_{e x} t\right) \\
& \left.-\left.S \mu_{0} \frac{\partial \mu_{r}}{\partial H}\right|_{H_{m}} \frac{\omega_{e x}}{2} h_{e x}{ }^{2} \sin \left(2 \omega_{e x} t\right)\right)
\end{aligned}
$$

where $S$ is the cross-sectional area of the sensing coil, $\omega_{e x}=2 \pi f_{e x}, \mu_{0}$ is the magnetic permeability in a vacuum and $\mu_{r}=\frac{1}{\mu_{0}} \frac{\partial \mathrm{B}}{\partial \mathrm{H}}$ the small signal magnetic permeability of the SPM material.

$\varepsilon(t)$ is composed by a term at $\omega_{e x}$ (first harmonic) and a term at $2 \omega_{e x}$ (second harmonic). The amplitude of the first harmonic is proportional to the first derivative of the magnetisation characteristic of the SPM material with respect to $H$ at $H_{m}$ (via $\mu_{r}=\left.\frac{1}{\mu_{0}} \frac{\partial \mathrm{B}}{\partial \mathrm{H}}\right|_{\mathrm{H}_{\mathrm{m}}}$ ) while the amplitude of the second harmonic is proportional to the second derivative of $B$ with respect to $H$ at $H_{m}$ (via $\left.\frac{1}{\mu_{0}} \frac{\partial \mu_{r}}{\partial \mathrm{H}}\right|_{\mathrm{H}_{\mathrm{m}}}$ ). Since $\frac{d B}{d H}$ is an even function, the determination of $H_{m}$ from the measurement of the first harmonic of the EMF would lead to an ambiguity on the sign of $H_{m}$. Conversely $\frac{d^{2} B}{d H^{2}}$ is an odd function that is monotonous over a large magnetic field range [7]. Consequently within this range, it is possible to determine $H_{m}$ without a sign ambiguity from the measurement of the second harmonic component of the EMF. Moreover, the first harmonic is maximum in the absence of a current to measure whereas the second harmonic is null. For these reasons the measurement of the second harmonic is recommended.

\section{B. Double core toroidal Neel effect sensor}

Let us consider a Neel effect current sensor implementing two coaxial toroidal SPM transducers, each of them featuring a winding, these windings being connected in series and wound in opposite direction with respect to each other (Fig. 1). The so obtained single coil is used both for the excitation of the transducer and the sensing. The excitation current is provided thanks to sinusoidal voltages of same amplitude but in phase opposition applied at both ends of the coil respectively (Fig.1). The output signal is measured at the middle point of the coil. The primary conductor fed by the current $I_{m}$ to measure is assumed be encircled by the sensor. According to (2) and assuming that the magnetic flux density is homogeneous on the surface of the windings, the EMF sensed by a torus reads:

$$
\begin{aligned}
e_{i}(t) \cong- & N S \mu_{0} \mu_{r}\left(H_{m}\right) \omega_{e x} h_{e x} \cos \left(\omega_{e x} t\right) \\
& \left.-\left.N S \mu_{0} \frac{\partial \mu_{r}}{\partial H}\right|_{H_{m}} \frac{\omega_{e x}}{2} h_{e x}{ }^{2} \sin \left(2 \omega_{e x} t\right)\right)
\end{aligned}
$$

where $\mathrm{i} \in\{1,2\}$ denotes the considered torus, $H_{m}$ is the amplitude of the magnetic field created by the current to measure $I_{m}, N$ is the number of turns on each torus.

The total EMF sensed at the middle point of the windings connected in series is

$$
e(t)=\frac{1}{2}\left(e_{1}(t)-e_{2}(t)\right)
$$

$e_{1}(t)$ and $e_{2}(t)$ both feature a component at $\omega_{e x}$ and a

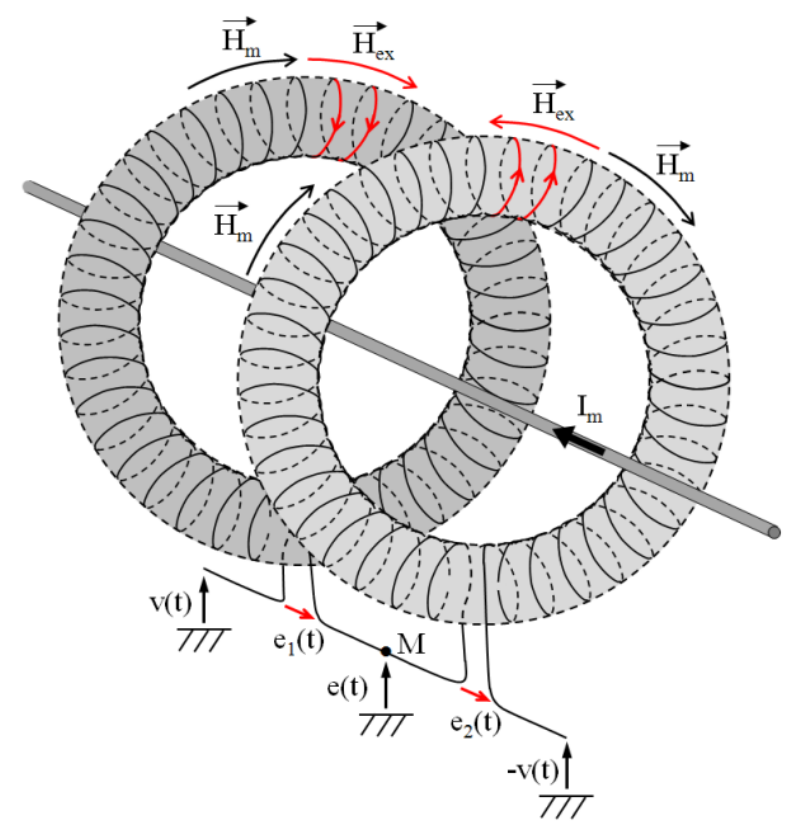

Fig. 1 Neel Effect sensor topology implementing two cores featuring homogeneous windings wound in opposite directions and connected in series.

component at $2 \omega_{e x}$. Since $\mu_{r}(H)$ is an even and $\frac{\partial \mu_{r}}{\partial H}$ an odd function [7], given the sensed magnetic field components directions (Fig. 1), the components of $e_{l}(t)$ and $-e_{2}(t)$ at $\omega_{e x}$ cancel each other out. Thus the sensed signal is

$$
e(t)=-\left.2 N S \mu_{0} \frac{\partial \mu_{r}}{\partial H}\right|_{H_{m}} \frac{\omega_{e x}}{2} h_{e x}{ }^{2} \sin \left(2 \omega_{e x} t\right)
$$

The proposed sensor configuration enables the cancellation of the first harmonic component, which makes the detection of the second harmonic component easier. Indeed, for Neel effect current sensors featuring a structure such that the first harmonic is not removed, the amplitude of the latter component is usually several orders higher than that of the second harmonic.

\section{Characterization OF A DEVICE}

A small size ring shaped Neel Effect sensor prototype implementing two SPM cores made up of a plastic matrix loaded with maghemite nanoparticles has been built by Neelogy. The inner and outer radii of the device are $15 \mathrm{~mm}$ and $30 \mathrm{~mm}$ respectively. Each core is homogeneously wound with 1400 turns of a copper wire of diameter $0.2 \mathrm{~mm}$. The inductance of one coil is $3,024 \mathrm{mH}$. According to the configuration described above, the coils are wound in opposite direction with respect to each other and connected in series. The latter device was characterized both for DC current and AC current measurement thanks to the experimental setup depicted in Fig. 2. The primary conductor crossed by the $I_{m}$ current to measure did consist in 20 turns of a copper wire of diameter $1 \mathrm{~mm}$ passing through the ring sensor. In this way, 


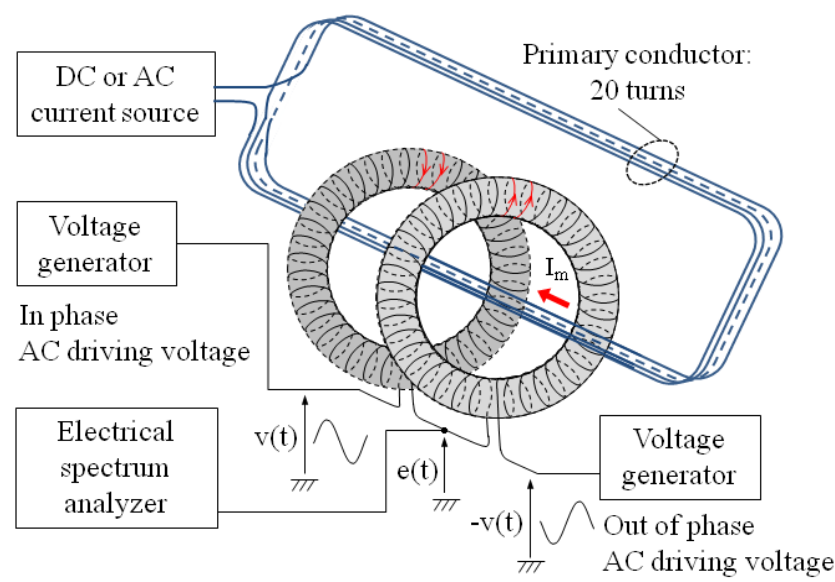

Fig. 2. Experimental setup for the characterization of a Neel Effect current sensor featuring a double core topology.

assuming the maximum current inside the wire to be in the order of $10 \mathrm{~A}$, the characterization of the sensor could be performed up to approximately 200 A. For the measurement of DC currents a 0-6 V/0-35 A DC power supply (Agilent $6574 \mathrm{~A}$ ) was connected to the primary conductor. The in phase and the out of phase excitation signals were provided by synchronized voltage generators connected at each end of the winding of the current sensor. The output EMF was measured at the middle of the winding by means of an electrical spectrum analyzer (Stanford research systems SR760FFT) featuring a $1 \mathrm{M} \Omega$ input impedance. With regards to the measurement of AC currents, the DC power supply was replaced by a $\mathrm{DC}-\mathrm{AC}$ converter capable of providing an $\mathrm{AC}$ current up to a $7 \mathrm{~A}$ amplitude.

The measurement of a DC current was carried out over the [-190 A $190 \mathrm{~A}]$ range and different excitation conditions were tested. Fig. 3 provides with the second harmonic component of the sensor output signal measured for an excitation current of fixed amplitude $I_{e x}=60 \mathrm{~mA}$ rms and for different excitation frequencies $f_{e x}$. Fig. 4 provides with the measures obtained at $f_{e x}=40 \mathrm{kHz}$ and for different excitation amplitudes. Firstly, it can be observed from the measurements that the amplitude of the output signal is in the order of a few $\mathrm{mV}$, which is consistent with theoretical and experimental results previously obtained [8]. Secondly, as the sensor is inductive the amplitude of the output signal increases as $f_{e x}$ increases (Fig. 3). Moreover, as $I_{e x}$ increases the sensitivity of the sensor also increases and, as shown in Fig. 5, within the $I_{m}$ range where the sensor response can be considered as relatively linear, this increase is proportional to the square of $I_{e x}$, which is consistent with (5) assuming that $h_{e x}$ is proportional to $I_{e x}$. As far as the first harmonic of the sensor output signal is concerned, the measured amplitudes were in the order of a few $\mathrm{mV}$ and variations lower than $10 \%$ over the whole considered $I_{m}$ measurement range were observed. This residual first harmonic component shall be attributed to imperfection of the symmetry of the windings of the two cores.

With regards to the measurement of an $\mathrm{AC}$ current, it was

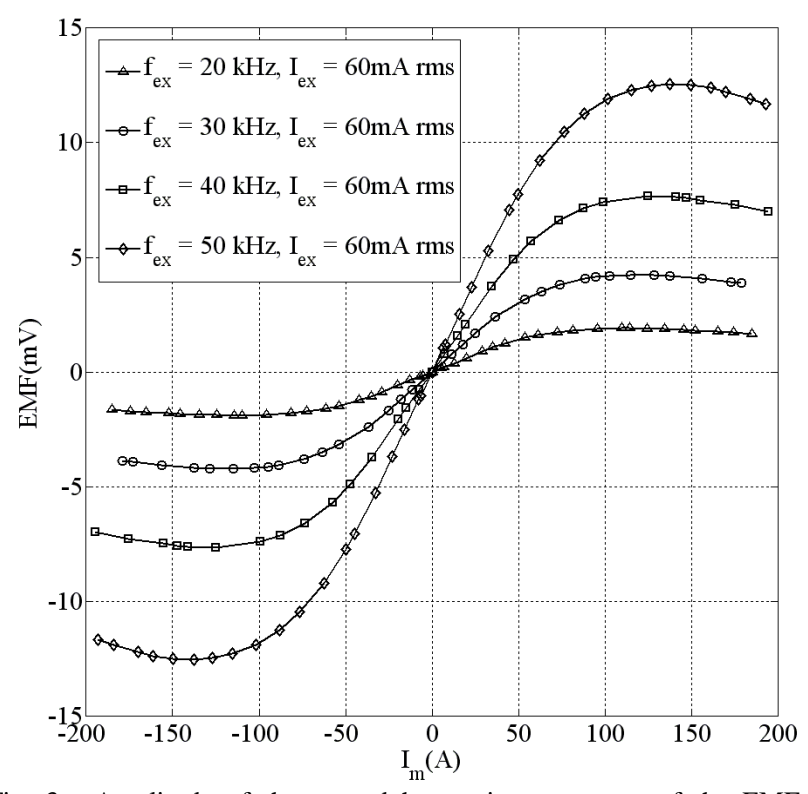

Fig. 3. Amplitude of the second harmonic component of the EMF measured at the sensor ouput as a function of the DC current to measure and for an excitation current of $60 \mathrm{~mA} \mathrm{rms}$ and for different excitation frequencies.

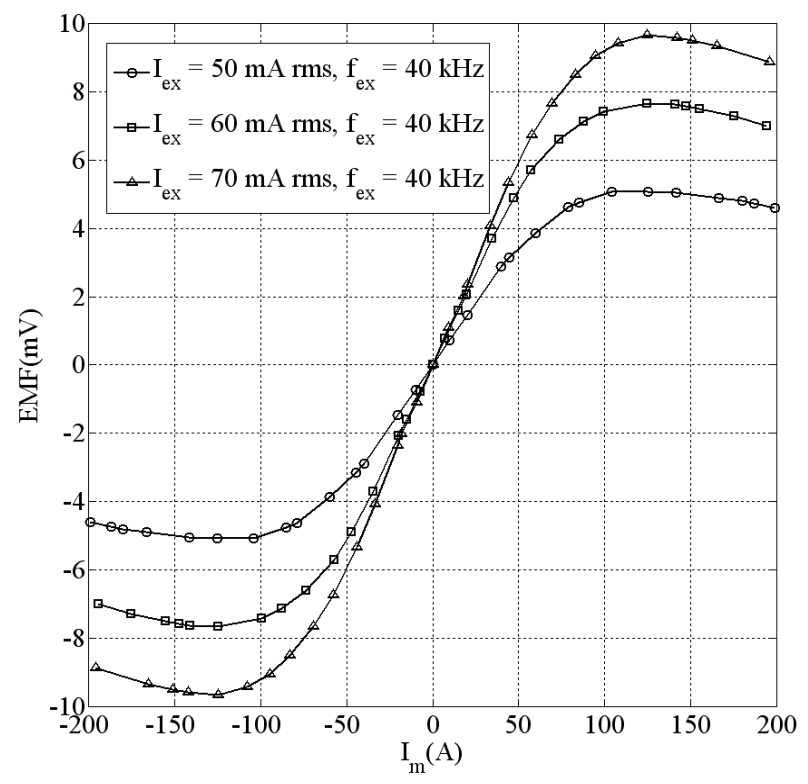

Fig. 4. Amplitude of the second harmonic component of the EMF measured at the sensor ouput as a function of the DC current to measure and for several amplitudes of the excitation current oscillating at $40 \mathrm{kHz}$.

carried out over the [-130 A $130 \mathrm{~A}]$ range for a current to measure of frequency $400 \mathrm{~Hz}$. Different excitation conditions were tested. Fig. 6 shows the second harmonic component of the sensor output signal measured for an excitation current of fixed amplitude $I_{e x}=60 \mathrm{~mA}$ rms and for different excitation frequencies $f_{e x}$. These results are consistent with those obtained for DC measured currents. It is to be noted that there exists a factor 2 between the results of Fig. 6 and Fig. 3. This factor originates from the fact that when measuring an $\mathrm{AC}$ 


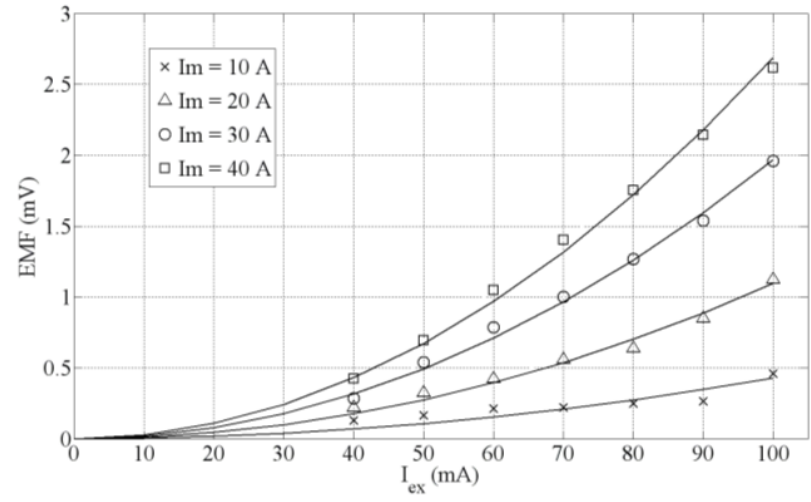

Fig. 5. Measured EMF second order harmonic plotted versus the amplitude of the $I_{e x}$ excitation current, for $f_{e x}=20 \mathrm{kHz}$, for different DC $I_{m}$ currents. The dashed lines are parabola extrapolated from the data.

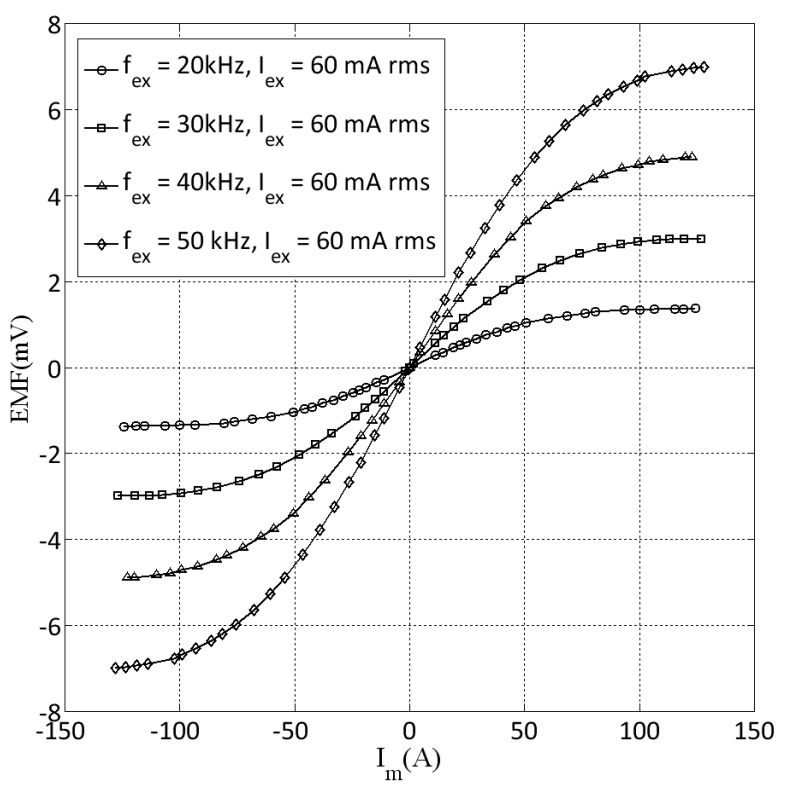

Fig. 6. Amplitude of the second harmonic component of the EMF measured at the sensor ouput as a function of the AC current to measure and for an excitation current of $60 \mathrm{~mA} \mathrm{rms}$ and for different excitation frequencies.

current the measured spectrum is double side band. The values reported in Fig 6 correspond to the amplitude of one sideband. For both DC and AC operation of the tested device in open loop configuration the measurement range is $[-110 \mathrm{~A} 110 \mathrm{~A}]$, which corresponds to the range where the second harmonic is monotonous. For an excitation current of amplitude $60 \mathrm{~mA}$ rms at $f_{e x}=50 \mathrm{kHz}$, the accuracy (defined as the standard deviation of the measures) was lower than $0.5 \%$ for $\left|I_{m}\right| \in[10 \mathrm{~A} 110 \mathrm{~A}]$ and lower than $2.5 \%$ for $\left|I_{m}\right| \in\left[\begin{array}{llll}1 & \mathrm{~A} & 10 \mathrm{~A}\end{array}\right]$.

\section{CONCLUSION}

A configuration has been proposed for enabling the cancellation of the first harmonic component at the output of a Neel effect current sensor. A prototype device has been characterized in open loop operation. The measurement of an AC current has been reported for the first time. Further works should be focused on closed loop operation of the sensor with a view to optimize the performances in particular in terms of linearity of the response and in terms of dynamic range.

\section{REFERENCES}

[1] P. Ripka, "Electric current sensors: a review", Meas. Sci. Technol., vol 21, 112001 (23pp), 2010.

[2] S. Ziegler, R.C. Woodward, H.H.C. Iu, L.J. Borle, "Current sensing techniques: A Review", IEEE Sensors Journal, vol. 9, (4), pp. 354-376, April 2009.

[3] A. Edelstein "Advances in magnetometry", J. Phys.: Condens. Matter., vol. 16, (6), 165217 (28pp), 2007.

[4] K. Iwanson, G. Sinapius, W. Hoornet, "Measuring current, voltage and power", Elsevier (Amsterdam), 1999.

[5] L. Lenglet 2007, "Current \& magnetic field sensors, control method \& magnetic core for said sensors", Billanco Patent WO2007042646 (A1).

[6] L. Néel, Ann. Grophys. (C.N.S.R.) 5,99, 1949.

[7] E. Vourc'h, P.-Y. Joubert, L. Cima, "Analytical and numerical analyses of a current sensor using non linear effects in a flexible magnetic transducer". Progress In Electromagnetics Research Journal, PIER 99, pp.323-338, 2009.

[8] E. Vourc'h, P.Y. Joubert, G. Cinquin, Y. Maniouloux, L. Cima, "Novel magnetic field and current sensors based on superparamagnetic transducers", Sensor Letters, volume 7, (3), pp. 293-298(6), June 2009.

Note: Neel Effect is a trade mark and a patented technology that belongs to Neelogy SA.

Corresponding author: E. Vourc'h (e-mail: eric.vourch@satie.ens-cachan.fr). 